\title{
gr-compactness in topological spaces
}

\section{K. Vithyasangaran ${ }^{1}$, J. Sriranganesan ${ }^{2}$ and S. Sathaananthan ${ }^{3}$}

Department of Mathematics, Faculty of Science, Eastern University, Sri Lanka

Type of Article: Research Paper

Received: 7 October 2021

Accepted: 12 November 2021

Online Ready: 24 November 2021

\begin{abstract}
In this paper, we introduce the new concepts gr-compactness in topological spaces and obtain some of their properties using gr-closed sets.
\end{abstract}

Keywords: gr-closed sets, gr-continuous maps and gr-compactness.

\section{Introduction}

The notions of compactness is useful and fundamental notions of not only general topology but also of other advanced branches of mathematics. Many researchers [1-7] have analyzed the basic properties of compactness. The notions of compactness resulted in motivating mathematicians to generalize these notions further.

Bhattacharya S. [8] introduced and studied the properties of gr-closed sets in topological spaces. The aim of this paper is to study gr-compactness using gr-closed set and also discuss some of their properties

\section{Preliminaries}

Throughout this paper $(X, \tau),(Y, \sigma)$ (or simply $X$ and $Y$ ) represent topological spaces on which no separation axioms are assumed unless otherwise mentioned. For a subset $A$ of $(X, \tau), \operatorname{cl}(A)$ and $\operatorname{Int}(A)$ denote the closure of $A$ and interior of $A$ respectively.

Definition 2.1. Let $(X, \tau)$ be a topological space. Then, a subset $A$ of $(X, \tau)$ is called gr-closed set [8] if $\operatorname{rcl}(A) \subseteq U$ whenever $A \subseteq U$ and $U$ is open in $(X, \tau)$.

The complement of the above mentioned gr-closed set is gr-open set.

Definition 2.2. A function $f:(X, \tau) \rightarrow(Y, \sigma)$ is called

(i) $g r$-continuous [9] if the inverse image of every closed set in $(Y, \sigma)$ is gr-closed in $(X, \tau)$.

(ii) $g r$-irresolute [9] if the inverse image of every gr-closed set in $(Y, \sigma)$ is gr-closed in $(X, \tau)$.

*Corresponding author: E-mail: vithyasangarank@esn.ac.lk 


\section{3 gr-compactness}

Definition 3.1. A collection $\left\{A_{i}: i \in l\right\}$ of gr-open sets in a topological space $X$ is called a gr-open cover of a subset $B$ of $X$ if $B \subset \cup\{A i: i \in l\}$ holds.

Definition 3.2. A topological space $X$ is gr-compact if every gr-open cover of $X$ has a finite subcover.

Definition 3.3. A subset $B$ of a topological space $X$ is said to be gr-compact relative to $X$ if, for every collection $\{A i: i \in I\}$ of gr-open subsets of $X$ such that $B \subset \cup\{A i: i \in I\}$ there exists a finite subset $I_{0}$ of $I$ such that $B \subseteq \cup\left\{A i: i \in I_{0}\right\}$.

Definition 3.4. A subset $B$ of a topological space $X$ is said to be $g r$-compact if $B$ is $g r$-compact as a subspace of $X$.

Theorem 3.1. Every gr-closed subset of a gr-compact space $X$ is gr-compact relative to $X$.

Proof. Let $A$ be $g r$-closed subset of $g r$-compact space $X$. Then, $A^{c}$ is $g r$-open in $X$. Let $M=\left\{G_{a}\right.$ : $\alpha \in I\}$ be a cover of $A$ by $g r$-open sets in $X$. Then, $M^{*}=M \cup A^{c}$ is a gr-open cover of $X$. Since $X$ is

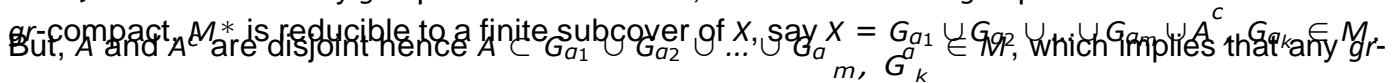
open cover $M$ of $A$ contains a finite subcover. Therefore, $A$ is $g r$-compact relative to $X$. Thus, every gr-closed subset of $g r$-compact space $X$ is $g r$-compact.

Theorem 3.2. Every gr-compact space is compact.

Proof. Let $X$ be a gr-compact space. Leł $A_{i}: i \in I$ \}be an open cover of $X$. Then $A_{i}: i \in I$ \}ंs a gr-open cover of $X$ as every open set is gr-open set. Since $X$ is gr-compact, the $g r$-open cover $\left\{A_{i}: i \in l\right\}$ of $X$ has a finite subcover, say $\left\{A_{i}: i=1, \ldots, n\right\}$ for $X$. Hence $X$ is compact.

Definition 3.5. A function $f: X \rightarrow Y$ is said to be gr-continuous [9] if $f^{-1}(F)$ is gr-closed in $X$ for every closed set $F$ of $Y$.

Definition 3.6. A function $f: X \rightarrow Y$ is said to be $g r$-irresolute [9] if $f^{-1}(F)$ is $g r$-closed in $X$ for every gr-closed set $F$ of $Y$.

Theorem 3.3. Letf $: X \rightarrow Y$ be surjective, gr-continuous function. If $X$ is gr-compact, then $Y$ is compact.

Proof. Let $\left\{A_{i}: i \in I\right\}$ be an open cover of $Y$. Since $f$ is gr-continuous function, then $\left\{f^{-1}\left(A_{i}\right)\right.$ : $i \in I\}$ is $g r$-open cover of $X$ has a finite subcover, say $\left\{f^{-1}\left(A_{i}\right): i=1, \ldots, n\right\}$. Therefore, $X=\cup{ }_{i=1}^{n} f^{-1}\left(A_{i}\right)$ which implies $f(X)=\cup_{i=1}^{n} f\left(A_{i}\right)$. Since $f$ is surjective, $Y=\cup_{i=1}^{n} f\left(A_{i}\right)$. Thus, $\left\{A_{1}, A_{2}, \ldots, A_{n}\right\}$ is a finite subcover of $\left\{A_{i}: i \in I\right\}$ for $Y$. Hence $Y$ is compact.

Theorem 3.4. If a map $f: X \not$ is gr-irresolute and a subset $B$ of $X$ is gr-compact relative to $X$, then the image $f(B)$ is gr-compact relative to $Y$.

Proof. Let $\left\{A_{a}: \alpha \in I\right\}$ be any collection of gr-open subsets of $Y$ such that $f(B) \subset \cup\left\{A_{a}: \alpha \in I\right\}$. Then, $B \subset\left\{f^{-1}\left(A_{a}\right): \alpha \in l\right\}$ holds. From the hypothesis, $B$ is gr-compact relative to $X$. Then, there exists a finite subset $I_{0}$ of $I$ such that $B \subset\left\{f^{-1}\left(A_{a}\right): \alpha \in I_{0}\right\}$. Therefore, we have $f(B) \subset \cup\left\{A_{a}\right.$ : $\alpha \in I_{0}$, which shows that $f(B)$ is gr-compact relative to $Y$.

\section{Conclusion}

In this paper, we have introduced gr-compactness in the topological spaces by using gr-closed sets and their properties were studied. 


\section{References}

[1] Hanif PAGE Md. and Hosamath V. T. A View on Compactness and Connectedness in Topological Spaces. Journal of Computer and Mathematical Sciences. 2019;10(6):1261-1268.

[2] Vivekannanda Dembre. and Sanjay M Mali. New Compactness and Connectedness in topological Spaces. International Journal of Applied Research. 2018;4(4):286-289.

[3] Rajeswari R. Darathi S. and Deva Margaret Helen D. Regular Strongly Compactness and Regular Strongly Connectedness in Topological Space. International Journal of Engineering Research and Technology (IJERT). 2020;9(2):547-550.

[4] Gnanambal Y. and Balachandran K. On gpr-continuous functions in topological spaces. Indian J. Pure Appl. Math. 1999;30(6):581-593.

[5] Pushpalatha A. Studies on Generalizations of Mappings in Topological Spaces. Ph.D. Thesis. Bharathiar University. Coimbatore. 2000.

[6] Sarika M. Patil and Rayanagoudar T. D. $\alpha g * s-C o m p a c t n e s s$ and $\alpha g * s-$ Connectedness in Topological Spaces. Global Journal of Pure and Applied Mathematics. 2017;13(7):3549-3559.

[7] Vivekananda Dembre and Pankaj B Gavali. Compactness and Connectedness in Weakly Topological Spaces. International Journal of Trend in Research and Development. 2018;5(2):606-608.

[8] Bhattacharya S. Study of On generalized regular closed sets. Int. J. Contemp. Math. Sciences. $2011 ; 6(3): 145-152$.

[9] Mahmood S. I. On Generalized Regular Continuous Functions In Topological Spaces. Ibn AlHaitham Journal for Pure and Applied Science. 2012;25(3):377-385. 